SVU- International Journal of Veterinary Sciences, 4 (4): 1-15, 2021.

Print ISSN: 2535-1826

\title{
Occurrence and characterization of coagulase positive and negative Staphylococci isolated from Japanese quails and broiler chickens at Qena Governorate, Egypt Waleed Younis ${ }^{1 *}$, Mahmoud Sabra², Haitham Helmy Sayed ${ }^{3}$
}

${ }^{1}$ Department of Microbiology, Faculty of Veterinary Medicine, South Valley University, Qena, 83523, Egypt, ${ }^{2}$ Department of Poultry Diseases, Faculty of Veterinary Medicine, South Valley University, Qena, 83523, Egypt, ${ }^{3}$ Department of Microbiology, Faculty of Veterinary Medicine, Sohag University, Sohag, 82524, Egypt.

\section{Abstract}

Staphylococcal infections in poultry are taking an increasing significance and both coagulase positive Staphylococci (CoPS) and coagulase negative Staphylococci (CoNS) can cause infections in poultry. On the other hand, methicillin resistance Staphylococci (MRS) is a global problem currently and MRS are often has multidrug resistance (MDR) against variety of the other antibiotics. This study was performed to investigate incidence of CoPS and CoNS and their characteristics among Japanese quails and broiler chickens in some farms at Qena Governorate Therefore, 80 Japanese quail samples of 3-4 weeks old and 70 broiler chicken samples of 21-25 days old were collected from different localities in Qena Governorate during the period from October 2020 to March 2021 for analysis. Bacterial isolates were identified as Staphylococci through phenotypic characteristics and PCR assay target 16s rRNA gene of Staphylococci, additionally CoPS were divided into Staphylococcus aureus (S. aureus) and other coagulase positive Staphylococci (OCoPS) by PCR assay target nuc gene of $S$. aureus. Bacteriologically, 11 Staphylococcus isolates were isolated from Japanese quail samples with percentage of $(13.75 \%)$ and they identified as $S$. aureus $(\mathrm{n}=2)$, OCoPS $(\mathrm{n}=2)$ and CoNS $(n=7)$ while 5 CoNS isolates were isolated and identified from broiler chicken samples with percentage of (7.14\%). Examination of Staphylococcus isolates for their ability to form biofilm by Microtiter plate (MTP) and Congo Red Agar (CRA) tests revealed that all isolates were biofilm producer but with varied grades of biofilm production and the correlation rate between MTP and CRA tests for detection of biofilm-producing Staphylococci were 93.75\%. Antimicrobial susceptibility testing of Staphylococcus isolates revealed that all the isolates were resistant to ampicillin, oxacillin, cefazolin, cefotaxime, vancomycin, erythromycin and clindamycin and they had antibiotic sensitivity to ciprofloxacin (87.5\%) followed by gentamicin (75.0\%), amoxicillin/clavulanic acid (62.5\%), oxytetracycline (25\%) and chloramphenicol (12.5\%). On the other hand, screening Staphylococcus isolates by PCR for presence of mecA, blaz and vanA resistance genes revealed that $(81.25 \%),(56.25 \%)$ and $(31.25 \%)$ of the isolates harbor these genes respectively and each MRS harbor one gene of mecA and blaz at least. The present study demonstrates that Japanese quails and chickens harbor multidrug-resistant bacteria that could be transmitted to human.

Keywords: Characterization, coagulase positive, coagulase negative, Staphylococci

DOI: 10.21608/svu.2021.92987.1146 Received: August 28, $2021 \quad$ Accepted: October 10, 2021

Published: October 21, 2021*Corresponding Authors:Waleed Younis E-mail: wkhrdr39@gmail.com Citation: Younis et al., Occurrence and characterization of coagulase positive and negative Staphylococci isolated from Japanese quails and broiler chickens at Qena Governorate, Egypt. SVUIJVS 2021, 4 (4): 1-15.

Copyright: () Younis et al. This is an open access article distributed under the terms of the creative common attribution license, which permits unrestricted use, distribution and reproduction in any medium provided the original author and source are created.

Competing interest: The authors have declared that no competing interest exists. 


\section{Introduction}

Poultry industry is considered one of the main sources of the national income in Egypt. Furthermore, it contributes to providing the population's needs of animal protein and achieving food security (Abdel Rahman and Abdel Motalib, 2018). Emergence and re-emergence of the diseases still to be the main challenges to the current situation and the strategic future of poultry industry (Hafez and Attia, 2020).

Staphylococci are one of the most common causes of infections in human, animals (Casey et al., 2007) and birds worldwide (Aarestrup et al., 2000), although, they are ubiquitous bacteria and are of the normal flora of skin, hair, nose and throat of human, animals (Anacarso et al., 2013) and poultry (Casey et al., 2007). Currently, genus Staphylococcus includes more than 70 species with presence of subspecies within some of these species (Götz et al., 2006). Based on their ability to clot plasma, Staphylococci are divided into two groups; CoNS and CoPS that include S. aureus (Anacarso et al., 2013).

S. aureus is the main pathogen within genus Staphylococcus, and it is one of the major pathogens associated with clinical infections and food poisoning in human as well as in animals all over the world (Mamza et al., 2019). Furthermore, CoNS have been responsible for multiple infections in both human and animals (Vuong and Otto, 2002). In poultry, most Staphylococcal infections are caused by CoPS especially $S$. aureus, however, CoNS have also been reported as pathogens in poultry infections (StępieńPyśniak et al., 2017).

Staphylococci are associated with many clinical infections in avian species worldwide (Onaolapo et al., 2017). These infections vary with site and route of infection in hatchery and poultry farms and include dermatitis, osteomyelitis, arthritis, synovitis, tenosynovitis, endocarditis, septicemia, omphalitis, femoral head necrosis and bumble foot which will in-turn causes large economic losses resulting from decreased weight gain, decreased egg production, lameness, mortality, and condemnation at slaughter of the birds (Lowder et al., 2009, Onaolapo et al., 2017 and Bakheet et al., 2018).

Antimicrobial resistance is an issue of increasing global concern and it is associated mainly with the uncontrolled usage of antimicrobials (Barber et al., 2003). In the recent years, Methicillin-resistant $S$. aureus (MRSA) has been growingly reported as emerging problem in the veterinary field especially in small animals and poultry (Broens et al., 2011). In addition, MRSA are often having MDR against a variety of the other antibiotics (Bakeet and Darwish, 2014). Moreover, there is a global resistance problem against vancomycin which is the last resort for the highly resistant $S$. aureus where vancomycin-resistant $S$. aureus (VRSA) has been isolated from different countries presently (Ammar et al., 2016). On the other hand, CoNS strains appear to be more resistant to antibiotics than $S$. aureus and MDR strains are observed with increasing frequency among this group (Marek et al., 2016). Development of multidrug resistant (MDR) pathogens makes prevention and control of the bacterial diseases of great difficulty affecting not only poultry but also human through transmission of such MDR pathogens and antibiotic resistance genes via consumption of the contaminated products (Darwish et al., 2013).

Biofilm formation is an important virulence and antibiotic resistance determinant. Biofilm producing S. aureus are widely distributed in poultry and poultry abattoirs in Egypt (Erfan and Marouf, 2015). Ability of CoNS to form biofilm structures on the damaged tissues surfaces is the main virulence factor in this bacterial group (de Silva et al., 2002). 
At present, there are limited researches on Staphylococcal infections in poultry and their antibiotic sensitivity in Egypt especially in quails and most these researches usually pertain to $S$. aureus although role of the other CoPS and CoNS in poultry infections as indicated in some literature data. On the other hand, as surge of pathogenic MRS, VRS and MDR Staphylococci continues in human, animals and poultry, there is imperative need for the continuous periodic monitoring of antibiotic susceptibility of Staphylococcus isolates for the effective treatment of Staphylococci infections. Therefore, this study was carried out to investigate occurrence of CoPS and CoNS among Japanese quails and broiler chickens in some farms at Qena Governorate and to determine their antimicrobial susceptibility profile including prevalence of MR, VR and MDR Staphylococci by both phenotypic and genotypic methods. Additionally, the current study aimed to determine ability of the isolates to form biofilm.

\section{Material and Methods}

\section{1- Sampling and clinical examination:}

During the period from October 2020 to March 2021, 80 Japanese quail samples of 34 weeks old and 70 broiler chicken samples of 21-25 days old were collected from different farms at different localities in Qena Governorate. They were subjected to clinical and post-mortem examinations at Poultry Diseases Department, Faculty of Veterinary Medicine, South Valley University, Egypt and samples were collected from liver, spleen, kidney and synovial fluid under aseptic conditions for bacteriological examination.

\section{2- Bacterial isolation and biochemical identification:}

Samples were inoculated into tryptone soya broth (TSB) (Oxoid, England) containing $70 \mathrm{mg} / \mathrm{ml} \mathrm{NaCl}$ and incubated at $37{ }^{\circ} \mathrm{C}$ under aerobic condition for $24 \mathrm{hrs}$. A loopful of the inoculated broth was subcultured on mannitol salt agar (Oxoid, England) and incubated at $37^{\circ} \mathrm{C}$ under aerobic condition for $24 \mathrm{hrs}$. The pink and yellow colonies of suspected isolates were preserved in TSB (Oxoid, England) containing $15 \%$ glycerol at $-20^{\circ} \mathrm{C}$ till further identification. Suspected isolates were tentatively identified according to the morphological characteristics, Gramstaining, coagulase, oxidase and catalase tests using the methods and criteria described by Quinn et al. (2004).

\section{3- Phenotypic characterization of biofilm formation:}

\section{1- Microtiter plate test:}

It was carried out as described by Melo et al. (2013) with slight modification. Briefly, the tested isolates were grown overnight in TSB (Oxoid, England) at $37^{\circ} \mathrm{C}$. Cultures were diluted by 1:200 with TSB (Oxoid, England) containing 1\% glucose (Oxford, India) then 200 $\mu$ l from each of these suspensions were placed in the wells of a 96-well polystyrene plate (Costar, USA), each isolate was tested in triplicate and three wells were used as a negative control containing $200 \mu \mathrm{l}$ of TSB and $1 \%$ glucose only. The plate was incubated at $37^{\circ} \mathrm{C}$ for 24 hrs. The bacterial suspensions were removed and the wells were washed three times by phosphate buffer saline, dried at $60{ }^{\circ} \mathrm{C}$ for 1 hr., stained with $0.5 \%$ crystal violet (Fluka, india) for $1 \mathrm{~min}$., washed three times by distilled water after removal of dye and finally dried at room temperature. Absorbance of the adherent biofilm (OD) was measured at $620 \mathrm{~nm}$ using microplate reader (BioTek ELX800, USA) and the results were interpreted according to Stepanović et al. (2007).

\section{2- Congo Red Agar test:}

It was carried out as described by Melo et al. (2013) where the tested isolates were grown on Brain heart infusion agar (Oxoid, UK) containing $0.08 \%$ Congo red (Oxford, 
India) and $3.6 \%$ sucrose (Oxford, India) at $37^{\circ} \mathrm{C}$ for $24 \mathrm{hrs}$. Results of CRA test were interpreted according to Dubravka et al. (2010) as follows:

a) Black colonies of dry consistency, rough surface and edges was considered biofilm producers.

b) Black colonies of smooth, round and shiny surface or red colonies of dry consistency, rough edges and surface was considered intermediate biofilm producers.

c) Red colonies of smooth, round and shiny surface were considered non-biofilm producers.

\section{4- Antimicrobial susceptibility testing:}

It was performed by the Kirby-Bauer disk diffusion method on Mueller-Hinton agar (MHA) according to Clinical and Laboratory Standards Institute guidelines (Wayne et al., 2008) .Briefly, the tested isolate was streaked onto MHA (Oxoid, UK), antibiotic disks were dispensed on the plate and the inoculated plate was incubated at $37^{\circ} \mathrm{C}$ for $24 \mathrm{hrs}$. Thereafter, inhibition zones diameters were measured and interpreted according to CLSI (2013). Twelve $(n=12)$ Antibiotics were chosen for this study according their common use for treatment of S. aureus infections, namely Ampicillin (AMP) (30 $\mu \mathrm{g})$, Amoxicillin/clavulanic acid (AMC) $(30 \mu \mathrm{g})$,

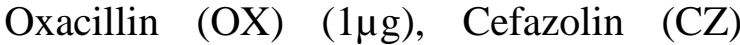

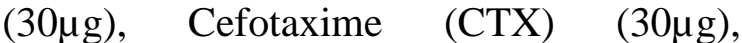

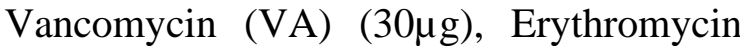
(E) $(15 \mu \mathrm{g})$, Gentamicin (CN) $(10 \mu \mathrm{g})$, Ciprofloxacin (CIP) $(5 \mu \mathrm{g})$, Oxytetracycline (T) $(30 \mu \mathrm{g})$, Chloramphenicol (C) $(30 \mu \mathrm{g})$ and Clindamycin (DA) $(2 \mu \mathrm{g})$ (HiMedia, India). Resistant to more than 3 antibiotics was considered as MDR (Magiorakos et al., 2012)

\section{5 - PCR for identification of Staphylococci and $S$. aureus as well as detection of some antibiotic resistance genes in the isolates:}

In this study, the isolates were genotypically identified as Staphylococci and as $S$. aureus by PCR assays targeting 16S rRNA gene of Staphylococci and nuc gene of $S$. aureus respectively. Furthermore, PCR assays were performed for detection of some antibiotic resistance genes in the isolates including mecA (a determinant of methicillin resistance), blaZ (a determinant of $\beta$-lactamase production) and vanA (a determinant of vancomycin resistance). The used oligonucleotide primers are illustrated in Table 1 and they were obtained from Metabion (Germany).

\section{1-DNA extraction:}

After growth of the tested isolates overnight in TSB (Oxoid, England) at $37^{\circ} \mathrm{C}$, bacterial DNA was extracted by QIAamp DNA Mini kit (Qiagen GmbH, Germany) according to manufacturer's instructions. Thereafter, extracted DNA concentrations were estimated by NanoDropTM Lite spectrometer (Thermo scientific, Germany) then it was preserved at $-20^{\circ} \mathrm{C}$ till be used.

\section{2-PCR amplification:}

DNA amplification was performed in Applied biosystem 2720 thermocycler (USA) using Emerald Amp Max PCR Master Mix (Takara, Japan) under PCR conditions illustrated in Table 1 for each target gene. According to master mix manufacturer's instructions, reaction mixture was performed in $25 \mu \mathrm{l}$ comprising Master Mix $(12.5 \mu \mathrm{l})$, forward and reverse primers ( $1 \mu \mathrm{l}$ from each), extracted DNA $(5 \mu \mathrm{l})$ and nuclease-free water $(5.5 \mu \mathrm{l})$.

\section{3-Analysis of PCR products:}

PCR products were electrophoresed on $1 \%$ agarose gel (Applichem $\mathrm{GmbH}$, Germany) in 1x TBE buffer. gene ruler 100 base pair (bp) DNA ladder (Thermo scientific, Germany) was used for fragments size determination. Thereafter, the gel was photographed by a gel documentation system (Alpha Innotech, Biometra). 


\begin{tabular}{|c|c|c|c|c|c|c|c|c|}
\hline \multirow{2}{*}{$\begin{array}{l}\text { Target } \\
\text { gene }\end{array}$} & \multirow{2}{*}{$\begin{array}{c}\text { Primers } \\
\text { sequences } \\
\left(5^{\prime}-3^{\prime}\right)\end{array}$} & \multirow{2}{*}{$\begin{array}{l}\text { Size } \\
\text { (bp) }\end{array}$} & \multirow{2}{*}{$\begin{array}{l}\text { Primary } \\
\text { Den. }\end{array}$} & \multicolumn{3}{|c|}{$\begin{array}{l}\text { PCR conditions } \\
\text { (35 cycles) }\end{array}$} & \multirow{2}{*}{$\begin{array}{c}\text { Final } \\
\text { extension }\end{array}$} & \multirow{2}{*}{ Ref. } \\
\hline & & & & Den. & Ann. & Ext. & & \\
\hline \multirow{2}{*}{$\begin{array}{l}\text { 16S } \\
\text { rRNA }\end{array}$} & $\begin{array}{l}\text { CCTATAAGACTGG } \\
\text { GATAACTTCGGG }\end{array}$ & \multirow{2}{*}{791} & \multirow{2}{*}{$\begin{array}{l}94^{\circ} \mathrm{C} \\
3 \mathrm{~min} .\end{array}$} & \multirow{2}{*}{$\begin{array}{c}94^{\circ} \mathrm{C} \\
90 \mathrm{sec} .\end{array}$} & \multirow{2}{*}{$\begin{array}{l}55^{\circ} \mathrm{C} \\
1 \mathrm{~min} .\end{array}$} & \multirow{2}{*}{$\begin{array}{l}72^{\circ} \mathrm{C} \\
1 \mathrm{~min} .\end{array}$} & \multirow{2}{*}{$\begin{array}{c}72^{\circ} \mathrm{C} \\
10 \mathrm{~min} .\end{array}$} & \multirow{2}{*}{$\begin{array}{c}\text { Mason et } \\
\text { al., } 2001\end{array}$} \\
\hline & $\begin{array}{l}\text { CTTTGAGTTTCAAC } \\
\text { CTTGCGGTCG }\end{array}$ & & & & & & & \\
\hline \multirow{2}{*}{ nuc } & $\begin{array}{l}\text { ATATGTATGGCAA } \\
\text { TCGTTTCAAT }\end{array}$ & \multirow{2}{*}{395} & \multirow{2}{*}{$\begin{array}{l}95^{\circ} \mathrm{C} \\
5 \mathrm{~min} .\end{array}$} & \multirow{2}{*}{$\begin{array}{c}95^{\circ} \mathrm{C} \\
30 \mathrm{sec} .\end{array}$} & \multirow{2}{*}{$\begin{array}{c}55^{\circ} \mathrm{C} \\
30 \mathrm{sec} .\end{array}$} & \multirow{2}{*}{$\begin{array}{c}72^{\circ} \mathrm{C} \\
30 \mathrm{sec} .\end{array}$} & \multirow{2}{*}{$\begin{array}{l}72^{\circ} \mathrm{C} \\
5 \mathrm{~min} .\end{array}$} & \multirow{2}{*}{$\begin{array}{c}\text { Gao et al. } \\
2011\end{array}$} \\
\hline & $\begin{array}{l}\text { GTAAATGCACTTG } \\
\text { CTTCAGGAC }\end{array}$ & & & & & & & \\
\hline \multirow{2}{*}{ mecA } & $\begin{array}{l}\text { GTAGAAATGACTG } \\
\text { AACGTCCGATAA }\end{array}$ & \multirow{2}{*}{310} & \multirow{2}{*}{$\begin{array}{l}94^{\circ} \mathrm{C} \\
5 \mathrm{~min} .\end{array}$} & \multirow{2}{*}{$\begin{array}{c}94^{\circ} \mathrm{C} \\
30 \mathrm{sec} .\end{array}$} & \multirow{2}{*}{$\begin{array}{c}50^{\circ} \mathrm{C} \\
30 \mathrm{sec} .\end{array}$} & \multirow{2}{*}{$\begin{array}{c}72^{\circ} \mathrm{C} \\
30 \mathrm{sec} .\end{array}$} & \multirow{2}{*}{$\begin{array}{l}72^{\circ} \mathrm{C} \\
7 \mathrm{~min} .\end{array}$} & \multirow{2}{*}{$\begin{array}{c}\text { McClure } \\
\text { et al., } \\
2006\end{array}$} \\
\hline & $\begin{array}{l}\text { CCAATTCCACATT } \\
\text { GTTTCGGTCTAA }\end{array}$ & & & & & & & \\
\hline \multirow{2}{*}{ blaZ } & $\begin{array}{c}\text { TACAACTGTAATA } \\
\text { TCGGAGGG }\end{array}$ & \multirow{2}{*}{833} & \multirow{2}{*}{$\begin{array}{l}94^{\circ} \mathrm{C} \\
5 \mathrm{~min} .\end{array}$} & \multirow{2}{*}{$\begin{array}{c}94^{\circ} \mathrm{C} \\
30 \mathrm{sec} .\end{array}$} & \multirow{2}{*}{$\begin{array}{l}50^{\circ} \mathrm{C} \\
40 \mathrm{sec} .\end{array}$} & \multirow{2}{*}{$\begin{array}{l}72^{\circ} \mathrm{C} \\
50 \mathrm{sec} .\end{array}$} & \multirow{2}{*}{$\begin{array}{l}72^{\circ} \mathrm{C} \\
10 \mathrm{~min} .\end{array}$} & \multirow{2}{*}{$\begin{array}{c}\text { Bagcigil } \\
\text { et al., } \\
2012\end{array}$} \\
\hline & $\begin{array}{l}\text { CATTACACTCTTG } \\
\text { GCGGTTTC }\end{array}$ & & & & & & & \\
\hline van $\mathrm{A}$ & $\begin{array}{c}\text { CATGACGTATCGG } \\
\text { TAAAATC } \\
\end{array}$ & 805 & $95^{\circ} \mathrm{C}$ & $94^{\circ} \mathrm{C}$ & $56^{\circ} \mathrm{C}$ & $74^{\circ} \mathrm{C}$ & $74^{\circ} \mathrm{C}$ & Patel et \\
\hline & $\begin{array}{c}\text { ACCGGGCAGRGTA } \\
\text { TTGAC }\end{array}$ & 883 & $10 \mathrm{~min}$. & $1 \mathrm{~min}$. & $1 \mathrm{~min}$. & $1 \mathrm{~min}$. & $10 \mathrm{~min}$. & al., 1997 \\
\hline
\end{tabular}

\section{Results}

\section{1-Results of clinical and post-mortem examination:}

In chickens, clinically, the infected broiler chickens showed lethargy, reluctance to move, lameness with swelling in the joints (Fig. 1A), dropping wing, some birds exhibited brownish watery diarrhea. Internally, liver appeared enlarged, friable with white to dull necrotic foci and some liver lobes had sharp demarcated areas consistent with an infarct (Fig.1B), spleen was also enlarged. Femoral head necrosis where the proximal growth plate separated from its articular cartridge.

On the other hand, the diseased Japanese quails showed clinically whitish to brownish diarrhea with lameness, bumble foot, foot bad dermatitis (Fig. 1C) and high mortality. Most observed post-mortem lesions were enlarged greenish liver with white necrotic patch (Fig. 1D). on the side, it must be mention that Salmonella serotypes and other Enterobacteriaceae were isolated from some cases at the same time from Japanese quails and chickens' samples (unpublished data).

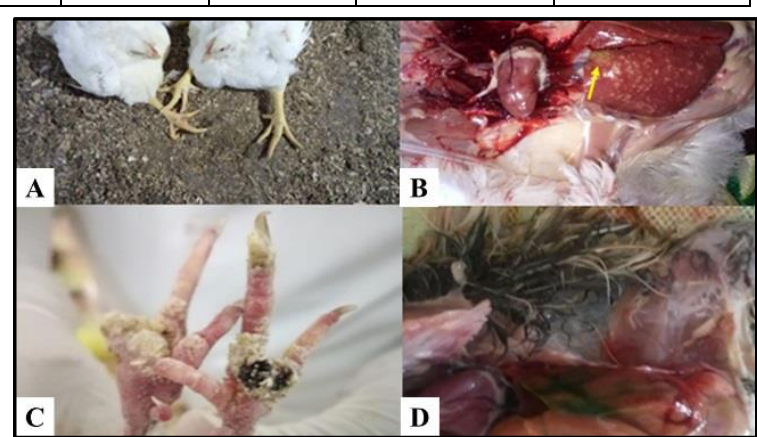

Fig. 1. Clinical and pathologic features of Staphylococci infection. A. Infected broiler chickens sits on the ground with their legs extended and unable to stand. B. Enlarged liver with white to dull necrotic foci and area of infarction (yellow arrow) in infected broiler chicken. C. Infected Japanese quail with bumble foot (pododermatitis). D. Enlarged greenish liver with white necrotic patch (yellow arrow) in infected Japanese quail.

\section{2- Results of bacterial isolation as well as} phenotypic and genotypic identification of the isolates:

Based on the morphological and biochemical characteristics of the isolates and their genotypic identification by PCR assays as illustrated in Fig. 2 and Fig. 3, 
incidence of Staphylococci, S. aureus, OCoPS and CoNS among the examined birds were summarized in Table 2 .

All Staphylococcus isolates produced yellow colonies on mannitol salt agar except three isolates produced pink colonies ( $\mathrm{S} 2$, S12 and S13). They were oxidase negative, catalase positive and coagulase negative except four isolates were coagulase positive (S1-S4).

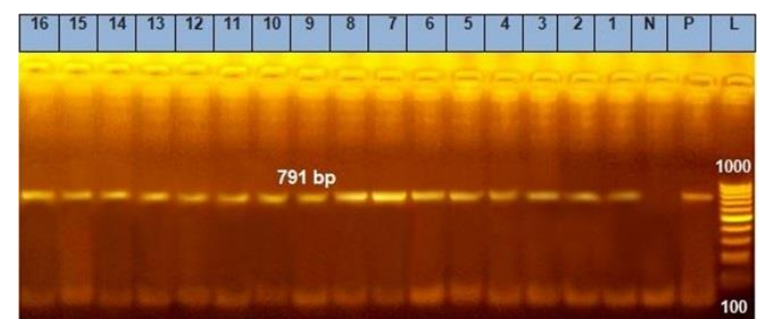

Fig. 2. Agar gel electrophoresis for PCR products using specific primers targeting 16s rRNA gene in Staphylococcus isolates. Lane L: 100 bp DNA ladder, lane P: positive control, lane N: negative control and lanes 116: PCR products from Staphylococcus isolates showing positive bands at 791-bp in all the isolates.

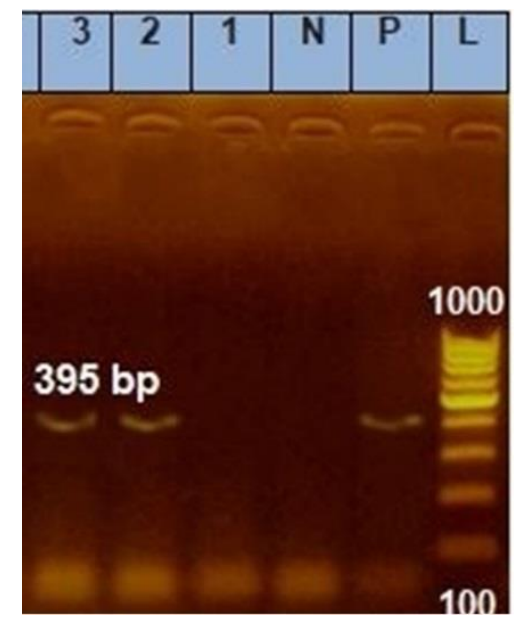

Fig. 3. Agar gel electrophoresis for PCR products using specific primers targeting nuc gene in CoPS isolates. Lane L: $100 \mathrm{bp}$ DNA ladder, lane P: positive control, lane N: negative control and lanes 1-4: PCR products from CoPS isolates (S1:S4 respectively) showing positive bands at 395 bp only in S2 and S3 isolates.

Table 2. Incidence of Staphylococci among the examined birds.

\begin{tabular}{|c|c|c|c|c|c|}
\hline \multirow{2}{*}{$\begin{array}{c}\text { Examined } \\
\text { birds }\end{array}$} & \multirow{2}{*}{$\begin{array}{c}\text { Examined } \\
\text { samples (No.) }\end{array}$} & $\begin{array}{c}\text { Staphylococcus } \\
\text { isolates (total) }\end{array}$ & \multicolumn{2}{|c|}{ CoPS isolates } & CoNS \\
\cline { 3 - 5 } & No. $(\%)$ & No. $(\%)$ & No. $(\%)$ & No. $(\%)$ \\
\hline Quails & 80 & $11(13.75 \%)$ & $2(2.5 \%)$ & $2(2.5 \%)$ & $7(8.75 \%)$ \\
\hline Chickens & 70 & $5(7.14 \%)$ & $0(0.0 \%)$ & $0(0.0 \%)$ & $5(7.14 \%)$ \\
\hline Total & ---- & $16(100.0 \%)$ & $2(12.5 \%)$ & $2(12.5 \%)$ & $12(75.0 \%)$ \\
\hline
\end{tabular}

\section{3- Results of biofilm formation:}

Results of biofilm formation of Staphylococcus isolates were summarized in Table 3. It was found that all the isolates were biofilm producer but with varied

Table 3. Results of biofilm formation by MTP and CRA tests.

\begin{tabular}{|c|c|c|c|c|c|c|c|c|c|}
\hline \multirow{3}{*}{ Test } & $\begin{array}{c}\text { Biofilm production } \\
\text { grade }\end{array}$ & \multicolumn{2}{c|}{$\begin{array}{c}\text { Staphylococci } \\
\text { isolates (total) }\end{array}$} & \multicolumn{2}{c|}{$\begin{array}{c}\text { S. aureus } \\
\text { isolates }\end{array}$} & \multicolumn{2}{c|}{$\begin{array}{c}\text { OCoPS } \\
\text { isolate }\end{array}$} & \multicolumn{2}{c|}{ CoNS isolates } \\
\cline { 3 - 10 } & & $\mathbf{N o .}$ & $\mathbf{\%}$ & $\mathbf{N o .}$ & $\mathbf{\%}$ & No. & $\mathbf{\%}$ & No. & $\%$ \\
\hline \multirow{3}{*}{ MTP } & Weak producer & 13 & 81.25 & 2 & 100.0 & 2 & 100.0 & 9 & 75.0 \\
\cline { 2 - 10 } & Moderate producer & 1 & 6.25 & 0 & 0.0 & 0 & 0.0 & 1 & 8.33 \\
\cline { 2 - 10 } & Strong producer & 2 & 12.5 & 0 & 0.0 & 0 & 0.0 & 2 & 16.67 \\
\hline \multirow{3}{*}{ CRA } & Biofilm producer & 3 & 18.75 & 1 & 50.0 & 0 & 0.0 & 2 & 16.67 \\
\cline { 2 - 10 } & Intermediate & 12 & 75.0 & 1 & 50.0 & 2 & 100.0 & 9 & 75.0 \\
\cline { 2 - 10 } & Non-biofilm & 1 & 6.25 & 0 & 0.0 & 0 & 0.0 & 1 & 8.33 \\
\hline
\end{tabular}




\section{4- Antimicrobial susceptibility testing:}

Results of evaluation antimicrobial susceptibility of Staphylococcus isolates to the tested antibiotics in this study were illustrated in Table 4. The results revealed that all Staphylococcus isolates were resistant to seven of the tested antibiotics namely ampicillin, oxacillin, cefazolin, cefotaxime, vancomycin, erythromycin and clindamycin. Concerning the other five antibiotics tested, the isolates had antibiotic sensitivity to ciprofloxacin (87.5\%) followed by gentamicin (75.0\%), amoxicillin/clavulanic acid (62.5\%), oxytetracycline $(25 \%)$ and chloramphenicol $(12.5 \%)$.

Table 4. Results of antimicrobial susceptibility of Staphylococcus isolates.

\begin{tabular}{|c|c|c|c|c|c|c|c|c|c|c|c|c|c|}
\hline \multirow{2}{*}{$\begin{array}{c}\text { Isolate } \\
\text { No. }\end{array}$} & \multicolumn{13}{|c|}{ Tested antibiotic } \\
\hline & AMP & AMC & $\mathbf{O X}$ & $\mathrm{CZ}$ & CTX & $\mathbf{V}$ & $\mathbf{E}$ & $\mathbf{C N}$ & CIP & $\mathbf{T}$ & $\mathbf{C}$ & DA & MDR \\
\hline \multicolumn{14}{|c|}{ S. aureus isolates (isolates from Japanese quails) } \\
\hline S2 & $\mathrm{R}$ & I & $\mathrm{R}$ & $\mathrm{R}$ & $\mathrm{R}$ & $\mathrm{R}$ & $\mathrm{R}$ & I & $\mathrm{R}$ & $\mathrm{R}$ & $\mathrm{R}$ & $\mathrm{R}$ & 10 \\
\hline S3 & $\mathrm{R}$ & $\mathrm{R}$ & $\mathrm{R}$ & $\mathrm{R}$ & $\mathrm{R}$ & $\mathrm{R}$ & $\mathrm{R}$ & $S$ & S & $\mathrm{R}$ & $\mathrm{R}$ & $\mathrm{R}$ & 10 \\
\hline \multicolumn{14}{|c|}{ Other CoPS isolates (isolated from Japanese quails) } \\
\hline S1 & $\mathrm{R}$ & $\mathrm{R}$ & $\mathrm{R}$ & $\mathrm{R}$ & $\mathrm{R}$ & $\mathrm{R}$ & $\mathrm{R}$ & I & $\mathrm{S}$ & $\mathrm{R}$ & $\mathrm{R}$ & $\mathrm{R}$ & 10 \\
\hline S4 & $\mathrm{R}$ & S & $\mathrm{R}$ & $\mathrm{R}$ & $\mathrm{R}$ & $\mathrm{R}$ & $\mathrm{R}$ & I & S & I & $\mathrm{R}$ & $\mathrm{R}$ & 8 \\
\hline \multicolumn{14}{|c|}{ CoNS isolates (isolated from Japanese quails) } \\
\hline S5 & $\mathrm{R}$ & $\mathrm{R}$ & $\mathrm{R}$ & $\mathrm{R}$ & $\mathrm{R}$ & $\mathrm{R}$ & $\mathrm{R}$ & $\mathrm{R}$ & I & $\mathrm{R}$ & $\mathrm{R}$ & $\mathrm{R}$ & 11 \\
\hline S6 & $\mathrm{R}$ & $\mathrm{R}$ & $\mathrm{R}$ & $\mathrm{R}$ & $\mathrm{R}$ & $\mathrm{R}$ & $\mathrm{R}$ & $\mathrm{S}$ & $\mathrm{R}$ & $\mathrm{R}$ & $\mathrm{R}$ & $\mathrm{R}$ & 11 \\
\hline S7 & $\mathrm{R}$ & I & $\mathrm{R}$ & $\mathrm{R}$ & $\mathrm{R}$ & $\mathrm{R}$ & $\mathrm{R}$ & $\mathrm{S}$ & I & I & $\mathrm{R}$ & $\mathrm{R}$ & 8 \\
\hline S8 & $\mathrm{R}$ & I & $\mathrm{R}$ & $\mathrm{R}$ & $\mathrm{R}$ & $\mathrm{R}$ & $\mathrm{R}$ & $\mathrm{S}$ & I & $\mathrm{R}$ & $\mathrm{R}$ & $\mathrm{R}$ & 9 \\
\hline S9 & $\mathrm{R}$ & I & $\mathrm{R}$ & $\mathrm{R}$ & $\mathrm{R}$ & $\mathrm{R}$ & $\mathrm{R}$ & $\mathrm{R}$ & $S$ & $\mathrm{R}$ & $\mathrm{R}$ & $\mathrm{R}$ & 10 \\
\hline S10 & $\mathrm{R}$ & I & $\mathrm{R}$ & $\mathrm{R}$ & $\mathrm{R}$ & $\mathrm{R}$ & $\mathrm{R}$ & $\mathrm{S}$ & I & $\mathrm{R}$ & $\mathrm{R}$ & $\mathrm{R}$ & 9 \\
\hline S11 & $\mathrm{R}$ & $\mathrm{S}$ & $\mathrm{R}$ & $\mathrm{R}$ & $\mathrm{R}$ & $\mathrm{R}$ & $\mathrm{R}$ & I & $\mathrm{S}$ & $\mathrm{R}$ & $\mathrm{R}$ & $\mathrm{R}$ & 9 \\
\hline \multicolumn{14}{|c|}{ CoNS isolates (isolated from broiler chickens) } \\
\hline S12 & $\mathrm{R}$ & $\mathrm{I}$ & $\mathrm{R}$ & $\mathrm{R}$ & $\mathrm{R}$ & $\mathrm{R}$ & $\mathrm{R}$ & $S$ & $S$ & I & $\mathrm{R}$ & $\mathrm{R}$ & 8 \\
\hline S13 & $\mathrm{R}$ & $\mathrm{R}$ & $\mathrm{R}$ & $\mathrm{R}$ & $\mathrm{R}$ & $\mathrm{R}$ & $\mathrm{R}$ & I & $S$ & $\mathrm{R}$ & $S$ & $\mathrm{R}$ & 9 \\
\hline S14 & $\mathrm{R}$ & $S$ & $\mathrm{R}$ & $\mathrm{R}$ & $\mathrm{R}$ & $\mathrm{R}$ & $\mathrm{R}$ & $\mathrm{I}$ & $\mathrm{I}$ & $\mathrm{S}$ & $\mathrm{S}$ & $\mathrm{R}$ & 7 \\
\hline S15 & $\mathrm{R}$ & $S$ & $\mathrm{R}$ & $\mathrm{R}$ & $\mathrm{R}$ & $\mathrm{R}$ & $\mathrm{R}$ & $\mathrm{R}$ & $S$ & $\mathrm{R}$ & $\mathrm{R}$ & $\mathrm{R}$ & 10 \\
\hline S16 & $\mathrm{R}$ & $\mathrm{R}$ & $\mathrm{R}$ & $\mathrm{R}$ & $\mathrm{R}$ & $\mathrm{R}$ & $\mathrm{R}$ & $\mathrm{R}$ & S & $\mathrm{R}$ & $\mathrm{R}$ & $\mathrm{R}$ & 11 \\
\hline $\begin{array}{c}\text { Sensitive } \\
\text { isolates }\end{array}$ & 0 & 10 & 0 & 0 & 0 & 0 & 0 & 12 & 14 & 4 & 2 & 0 & \\
\hline$\%$ & 0 & 62.5 & 0 & 0 & 0 & 0 & 0 & 75.0 & 87.5 & 25.0 & 12.5 & 0 & \\
\hline
\end{tabular}

$\mathrm{R}=$ resistant, $\mathrm{S}=$ sensitive and $\mathrm{I}=$ intermediate.

\section{5- Isolates investigation for some antibiotic resistance genes by PCR:}

Results of screening of Staphylococcus isolates by conventional PCR for presence of mecA, blaZ and vanA genes were summarized in Table 5 and illustrated in Figs. 4., Fig. 5, and Fig. 6 respectively.

Relationship between phenotypic MR and presence of mecA and blaZ genes in the isolates were summarized in Table 6.

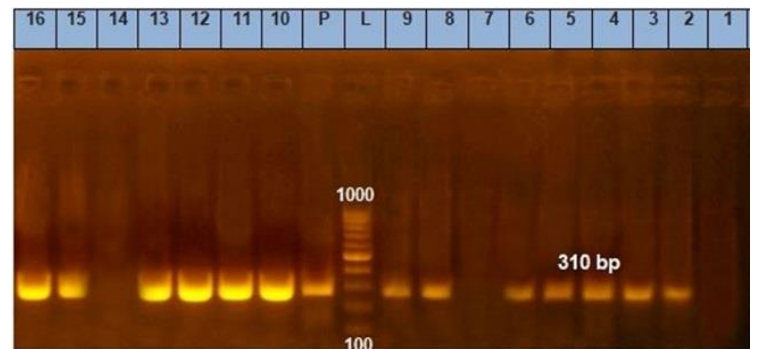

Fig. 4. Agar gel electrophoresis for PCR products using specific primers targeting mecA gene in Staphylococcus isolates. Lane L: 100 bp DNA ladder, lane P: positive control, lane N: negative control and lane 1-16: PCR products from Staphylococcus isolates (S1:S16 
respectively) showing positive bands at 310-bp in all the isolates except S1, S7 and S14.

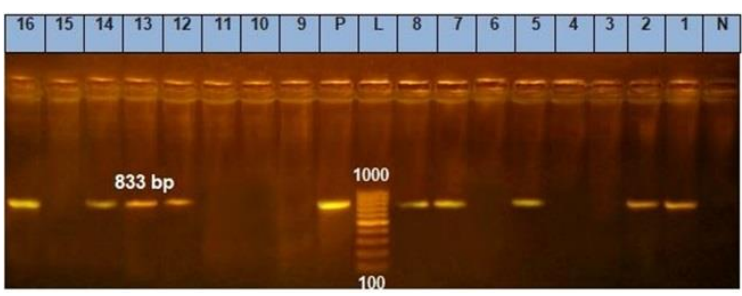

Fig. 5. Agar gel electrophoresis of the PCR products using specific primers targeting blaZ gene in Staphylococcus isolates. Lane L: 100 bp DNA ladder, lane P: positive control, lane N: negative control and lanes 1-16: PCR products from Staphylococcus isolates (S1:S16 respectively) showing positive bands at 833-bp in all the isolates except S3, S4, S6, S9, S10, S11 and $\mathrm{S} 15$.

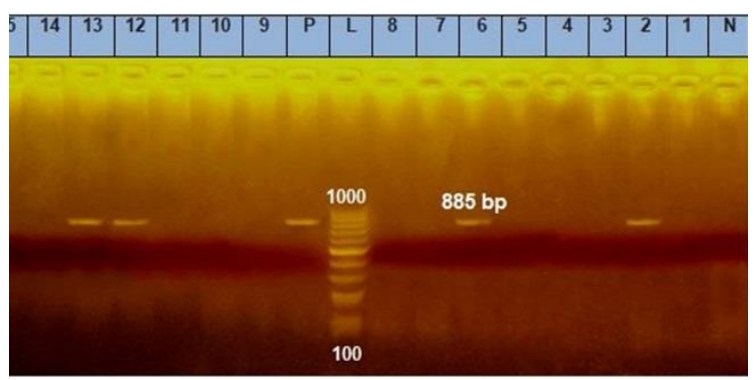

Fig. 6. Agar gel electrophoresis for PCR products using specific primers target vanA gene in Staphylococcus isolates. Lane L: 100 bp DNA ladder, lane P: positive control, lane N: negative control and lanes 1-16: PCR products from Staphylococcus isolates (S1:S16 respectively) showing positive bands at 885-bp only in S2, S6, S12, S13 and S16 isolates.

Table 5. Prevalence of mecA, blaZ and vanA genes among Staphylococcus isolates by PCR.

\begin{tabular}{|c|c|c|c|c|c|c|c|c|c|}
\hline \multirow{2}{*}{ Gene } & \multirow{2}{*}{$\begin{array}{l}\text { Result of gene } \\
\text { detection in the } \\
\text { isolate }\end{array}$} & \multicolumn{2}{|c|}{$\begin{array}{l}\text { Staphylococcus } \\
\text { isolates (total) }\end{array}$} & \multicolumn{2}{|c|}{$\begin{array}{l}\text { S. aureus } \\
\text { isolates }\end{array}$} & \multicolumn{2}{|c|}{$\begin{array}{c}\text { OCoPS } \\
\text { isolates }\end{array}$} & \multicolumn{2}{|c|}{ CoNS isolates } \\
\hline & & No. & $\%$ & No. & $\%$ & No. & $\%$ & No. & $\%$ \\
\hline \multirow{2}{*}{ mecA } & Positive & 13 & 81.25 & 2 & 100 & 1 & 50.0 & 10 & 83.3 \\
\hline & Negative & 3 & 18.75 & 0 & 0.0 & 1 & 50.0 & 2 & 16.7 \\
\hline \multirow{2}{*}{ blaZ } & Positive & 9 & 56.25 & 1 & 50.0 & 1 & 50.0 & 7 & 58.3 \\
\hline & Negative & 7 & 43.75 & 1 & 50.0 & 1 & 50.0 & 5 & 41.7 \\
\hline \multirow{2}{*}{ vanA } & Positive & 5 & 31.25 & 1 & 50.0 & 0 & 0.0 & 4 & 33.3 \\
\hline & Negative & 11 & 68.75 & 1 & 50.0 & 2 & 100 & 8 & 66.7 \\
\hline
\end{tabular}

Table 6. Presence of $m e c A$ and/or blaZ genes among MR Staphylococcus isolates.

\begin{tabular}{|c|c|c|c|}
\hline & mecA gene & blaZ gene & Both mecA and blaZ gene \\
\hline Isolates No. & $\mathrm{S} 3, \mathrm{~S} 4, \mathrm{~S} 6, \mathrm{~S} 9, \mathrm{~S} 10, \mathrm{~S} 11$ and S15 & $\mathrm{S} 1, \mathrm{~S} 7$ and S14 & S2, S5, S8, S12, S13 and S16 \\
\hline Positive isolates No. & 7 & 3 & 6 \\
\hline$\%$ & $43.75 \%$ & $18.75 \%$ & $37.5 \%$ \\
\hline
\end{tabular}

\section{Discussion}

Staphylococcal infections in poultry are taking increasing significance. Although, $S$. aureus is the most frequently isolated species from poultry, the literature data indicate that other CoPS and CoNS can also cause infections in poultry and there is increase in role of CoNS in poultry infections which suggests that the safety risk associated with their occurrence in the clinical environment and food may be higher than previously thought (Marek et al., 2016). In this study and as illustrated in Table 2, it was found that incidence of Staphylococci (total), S. aureus, OCoPS and CoNS among the examined quails was $(13.75 \%),(2.5 \%)$, $(2.5 \%)$ and $(8.75 \%)$ respectively while their incidence among the examined chickens was (7.14\%), (0.0\%), (0.0\%) and (7.14\%) respectively. Our results came in accordance with findings of El-Jakee et al. (2008) who reported that incidence of Staphylococci among chickens was (12\%) while disagreed with those of Bakeet and Darwish (2014) and Salah et al. (2020) who reported that incidence of Staphylococci among chickens 
was $(72.5 \%)$ and $(35.0 \%)$ respectively and Shokry et al. (2018) who reported that Staphylococcus were isolated from diseased chickens by $69.23 \%$ and $38.95 \%$ from apparently healthy flocks . Our results came also in accordance with findings of Otalu et al. (2011) and Otalu et al. (2015) who reported that incidence of $S$. aureus among live and slaughtered chickens was $(3.25 \%)$ and $(0.93 \%)$ respectively while disagreed with those of Mamza et al. (2019) and Salah et al. (2020) who reported that incidence of $\mathrm{S}$. aureus among chickens was $(47.5 \%)$ and $(14.2 \%)$ respectively. Furthermore, in contrast to our results, very higher incidence of CoPS (42.5\%) were recorded among chickens by Bakeet and Darwish (2014) and higher incidence of CoNS (19.33\%) and $(25.7 \%)$ were recorded among chickens by El-Nagar et al. (2017) and Kawano et al. (1996). Non-hemolytic, coagulase-negative Staphylococcus was isolated from Culture of eyelid skin of Japanese quail (Raidal, 1995) and MRSA were isolated by $29 \%$ from One hundred swab samples from quails at the slaughterhouse Vanessa et al. (2021). These discrepancies in incidence of Staphylococcus spp. may be attributed to the differences in geographical location, management practices, numbers of samples, sampling methods and examination methods (Mamza et al., 2019 and Salah et al., 2020). Isolation of high percentage of CoNS $(75 \%)$ and OCoPS (12.5\%) from quails and chickens in this study confirm that CoNS and OCoPS are considered important poultry pathogens especially where they carry antimicrobial resistance genes as it was found also in our study and thus poultry can serve as a potential source or reservoirs of these pathogens transfer to humans and animals.

The clinical signs and post-mortem lesions observed in the infected broiler chickens and Japanese quails in this study agreed with those of Amen et al. (2019) who reported that chickens, turkeys and ducks infected with Staphylococci exhibited depression, wings dropping, dullness, inability to stand, lameness with enlargement of liver and spleen. Also, it agreed with those of Awan and Matsumoto (1998) who reported that Staphylococcal infection causes diarrhea, osteomyelitis, synovitis and/or bumble foot in the infected birds. On the other hand, our results disagreed with those of Popy et al. (2011) who reported clinical signs and post-mortem lesions in the Staphylococcal infected birds weren't observed in this study and which included oculo-nasal discharge, conjunctivitis, facial oedema, respiratory rales, catarrhal tracheitis and rhinitis.

MTP and CRA tests are commonly used for phenotypic identification of biofilmproducing bacteria (Erfan and Marouf, 2015). In this study, it was found that all the isolates were biofilm producer but with varied grades of biofilm production indicating their virulence. On the other hand, it was found that the correlation rate between MTP and CRA tests for detection of biofilmproducing Staphylococci were 93.75\%. This result agreed with that of Erfan and Marouf (2015) who reported that this correlation rate was $94.02 \%$ while contradicts that of Nasr et al. (2012) who reported that this correlation rate was $20 \%$.

In the recent years, use of antibiotics in poultry production has increased extensively (Pyzik et al., 2019) and some bacteria have demonstrated full or partial resistance to the different antibiotics (Palanisamy and Bamaiyi, 2015). As shown in Table 4, antimicrobial susceptibility testing of Staphylococcus isolates in the present study revealed surprisingly high resistance where all the isolates were resistant to seven of the tested antibiotics (MDR) namely ampicillin, oxacillin, cefazolin, cefotaxime, vancomycin, erythromycin and clindamycin and they had antibiotic sensitivity to ciprofloxacin (87.5\%) followed by gentamicin (75.0\%), amoxicillin/clavulanic acid $(62.5 \%)$, oxytetracycline $(25.0 \%)$ and chloramphenicol (12.5\%). Otalu et al. 
(2011), Marek et al. (2016), Onaolapo et al. (2017), Mamza et al. (2019), Mohamed et al. (2020) and Salah et al. (2020) reported that MDR Staphylococcus strains from chickens but with comparatively different patterns of resistance and sensitivity to the different antibiotics and which could be attributed to numerous differences including geographical location, susceptibility testing methodologies, antimicrobial usage, management practices (White et al., 2003) and time of examination where antimicrobial resistance percentage is increasing over time (Ali et al., 2017).

The high MDR observed in this study could be attributed to the abuse of these antibiotics in poultry farms in area of the study from a long time (Onaolapo et al., 2017) where there is no drug control or legislations concerning sales of antibiotics. On the other hand, the relatively low parentage of resistance against amoxicillin/clavulanic acid observed in this study could be attributed to action of beta lactamase inhibitor; clavulanic acid where these isolates were found to be resistant to $\beta$ lactam antibiotics. Also, parenteral administration of gentamicin which involve specialized hand for injection may have curtailed their abused in area of the study and subsequently the isolates showed high sensitivity to it (Onaolapo et al., 2017). From a clinical perspective, presence of such MDR bacteria will result in difficulty of Staphylococcal infections treatment in poultry due to the limited therapeutic options especially from the antibiotics commonly used for their treatment. This in addition to their epidemiological and public health implications represented in transfer of these MDR bacteria and their resistance genes to human, poultry and animals.

Currently, MRS is a global problem (Pyzik et al., 2019) and its percentage is increasing in an alarming rate (Ali et al., 2017). There are two mechanisms for methicillin resistance in Staphylococci, the first is associated with presence of mecA gene on the bacterial chromosome which encodes altered penicillin- binding proteins 2a (PBP2a) has a very low affinity to $\beta$ lactam antibiotics (Bakheet et al., 2018). As illustrated in Table 5 and Fig. 4, screening Staphylococcus isolates by PCR for presence of mecA gene revealed its presence in Staphylococi, S. aureus, OCoPS isolates and CoNS isolates with percentage $(81.25 \%)$, (100.0\%), (50.0\%) and (83.3\%) respectively. Our results agreed with those of Bakeet and Darwish (2014) and Salah et al. (2020) who found that all S. aureus isolates and $(80 \%)$ of CoNS isolates harbor mecA gene respectively. Bakeet and Darwish (2014) and Bakheet et al. (2018) detected mecA gene in OCoPS isolates with higher prevalence, $(62.5 \%)$ and $(72.7 \%)$ respectively while Pyzik et al. (2019) detected mecA gene in CoNS isolates with very lower prevalence $(27.6 \%)$. Prevalence of $m e c A$ gene with high percentage in our isolates could explain the MDR observed in such isolates where mecA gene complex allows the cross resistance to the other antibiotics because it carries insertion sites for mobile genetic elements that facilitate acquisition of resistance determinants to the other antibiotics (Bakheet et al., 2018 and Mohamed et al., 2020).

The second mechanism for $M R$ in Staphylococci is production of $\beta$-lactamase enzymes inactivate the antibiotic by hydrolysis of its $\beta$-lactam ring, these enzymes are encoded by blaZ gene located on the bacterial chromosome or plasmids (Bakheet et al., 2018). As illustrated in Table 5 and Fig. 5, screening Staphylococcus isolates by PCR for presence of blaZ gene revealed its presence in Staphylococci, $S$. aureus, OCoPS isolates and CoNS isolates with percentage $(56.25 \%),(50.0 \%),(50.0 \%)$ and $(58.3 \%)$ respectively. Our results agreed with those of Bakeet and Darwish (2014) and Pyzik et al. (2019) who found that $(45.45 \%)$ of OCoPS isolates and $(58.3 \%)$ of CoNS isolates harbor blaZ gene respectively while disagreed with those of Ferreira et al. 
(2017) and Salah et al. (2020) who found that $(82 \%)$ of Staphylococci isolates and all $S$. aureus isolates harbor blaZ gene respectively. In this study, it was found that prevalence of MR among Staphylococcus isolates was $100 \%$ and on comparing results of phenotypic prediction of mecA and blaZ genes and those of PCR assays, it was found that each MR isolate harbor one gene of $m e c A$ and blaZ genes at least where it was found that $(37.5 \%),(43.75 \%)$ and $(18.75 \%)$ of the isolates harbor both mecA and blaZ, $m e c A$ only and blaZ only respectively as illustrated in Table 6.

Presently, VRSA has been isolated from different countries (Ammar et al., 2016) where it causes a wide range of the infections in different hosts (Grundmann et al., 2010). As illustrated in Table 5 and Fig. 6, screening Staphylococcus isolates by PCR for presence of vanA gene responsible for depressing the cell wall affinity for vancomycin revealed its presence in Staphylococci, S. aureus, OCoPS isolates and CoNS isolates with percentage $(31.25 \%),(50.0 \%),(0.0 \%)$ and $(33.3 \%)$ respectively. Our results disagreed with those of Salah et al. (2020) who found that prevalence of vanA gene among Staphylococci, S. aureus and CoNS isolates was $(60.0 \%),(50.0 \%)$ and $(0.0 \%)$ respectively. In this study, it was found that all the isolates were resistant to vancomycin although only $(31.25 \%)$ of the isolates harbor vanA gene, this may be attributed to that vancomycin resistance may by expressed phenotypically through another resistance genes aren't tested in this study. From a clinical perspective, resistance of all Staphylococci isolates to vancomycin observed in this study gives us an alarm about the dangerous situation of Staphylococci resistance where vancomycin is the drug of last resort for highly drug resistant S. aureus (Ammar et al., 2016).

\section{Conclusion}

This study provided a foresight about incidence of Staphylococci among Japanese quails and broiler chickens in Qena Governorate, Egypt and their antibiotics susceptibility. It revealed that both CoPS and CoNS are important poultry pathogens and there is increase in role of CoNS in poultry infections. It also revealed an alarming level of Staphylococci resistance to many of the antibiotics commonly used for treatment of Staphylococcal infections in human and poultry and the high prevalence of $m e c A$, blaz and vanA resistance genes among Staphylococci isolates. Therefore, the immediate restriction for the indiscriminate use of antibiotics in poultry farms is very crucial with application of the sanitary hygienic measures in poultry production. Furthermore, there is a need for performing similar studies in the other areas of Egypt to obtain more comprehensive data and for monitoring susceptibility of the clinical isolates for the antibiotics periodically.

\section{Author's contributions}

All authors contributed equally in this work. They read and approved the final manuscript.

\section{Conflicts of interest}

The authors declare that they have no conflict of interest.

\section{References}

Aarestrup FM, Agers LY, Ahrens P, Jørgensen JCØ, Madsen M, Jensen LB (2000). Antimicrobial susceptibility and presence of resistance genes in Staphylococci from poultry. Veterinary Microbiology, 74 (4): 353364.

Abdel Rahman EA and Abdel motalib MA (2018). An economic study of production import and protection policies for poultry in Egypt. Egyptian Journal of Agricultural Research, 96 (4): 1653-1688.

Ali Y, Islam MA, Muzahid NH, Faruk SM, Hossain MA, Marzan LW (2017). 
Characterization, prevalence and antibiogram study of Staphylococcus aureus in poultry. Asian Pacific Journal of Tropical Biomedicine, 7(3): 253-256.

Amen O, Hussein AG, Sayed AM, Ibrahim RS (2019). Detection of antibiotics resistance genes in Staphylococcus aureus isolated from poultry farms. Assiut Veterinary Medical Journal, 65 (163):1-9.

Ammar AM, Attia AM, Abd El-Aziz NK, Abd El Hamid MI, El-Demerdash AS (2016). Class 1 integron and associated gene cassettes mediating multiple-drug resistance in some food borne pathogens. International Food Research Journal, 23(1): 332-339.

Anacarso I, Condò C, Sabia C, Messi P, Niederhausern S, Bondi M, Iseppi R (2013). Antimicrobial resistance and other related virulence factors in Staphylococcus Spp. isolated from food, environmental and humans in Italy. Universal Journal of Microbiology Research, 1(1): 1-9.

Awan MA and Matsumoto M (1998). Heterogeneity of Staphylococci and other bacteria isolated from six-weekold broiler chickens. Poultry Science, 77: 944-949.

Bagcigil AF, Taponen S, Koort J, Bengtsson B, Myllyniemi A-L, Pyörälä S (2012). Genetic basis of penicillin resistance of $S$. aureus isolated in bovine mastitis. Acta Veterinaria Scandinavica, 54:69.

Bakeet AA and Darwish SF (2014). Phenotypic and genotypic detection of methicillin-resistant Staphylococcus aureus (MRSA) in broiler chickens. Assiut Veterinary Medical Journal, 60 (143): 142-151.
Bakheet AA, Amen O, AL Habaty SH, Darwish SF (2018). Prevalence of Staphylococcus aureus in broiler chickens with special reference to beta-lactam resistance genes in the isolated strains. Alexandria Journal of Veterinary Sciences, 57 (2): 25-33.

Barber DA, Miller GY, McNamara PE (2003). Models of antimicrobial resistance and foodborne illness: Examining assumptions and practical applications. Journal of Food Protection, 66:700-709.

Broens EM, Great EA, Van Der Wolf PJ, Van De Giesen AW, De Jong MC (2011). Prevalence and risk factor analysis of livestock associated MRSA-positive pig herds in The Netherlands. Preventive Veterinary Medicine, 102:41-49.

Casey AL, Lambert PA, Elliot TS (2007). Staphylococci. International Journal of Antimicrobial Agents, 29: 23-32.

CLSI (2013). Performance Standards for Antimicrobial Disk and Dilution Susceptibility Tests for Bacteria isolated from Animal, $4^{\text {th }} \mathrm{Ed}$. CLSI document Vet01-A4. Wayne, PA: Clinical and Laboratory Standards Institute, 33 (7).

Darwish WS, Eldaly E, El-Abbasy M, Ikenaka Y, Nakayama S, Ishizuka, M (2013). Antibiotic residues in food: African scenario. Japanese Journal of Veterinary Research, 61: S13-S22.

De Silva GD, Kantzanou M, Justice A, Massey RC, Wilkinson AR, Day NP, Peacock SJ (2002). The ica operon and biofilm production in coagulasenegative Staphylococci associated with carriage and disease in a neonatal intensive care unit. Journal of Clinical Microbiology, 40: 382-388. 
Dubravka M, Lazic S, Branka V, Jelena P, Bugarski D, Zorica S (2010). Slime production and biofilm forming ability by Staphylococcus aureus bovine mastitis isolates. Acta Veterinaria, 60 (2-3): 217-226.

El-Jakee J, Nagwa AS, Bakry M, Zouelfakar SA, Elgabry $\mathrm{E}$ and El-Said WG (2008). Characteristics of Staphylococcus aureus strains isolated from human and animal sources. American-Eurasian Journal of Agricultural \& Environmental Sciences, 4: 221-229.

El-Nagar S, Abd El-Azeem MW, Nasef SA, Sultan S (2017). Prevalence of toxigenic and methicillin resistant Staphylococci in poultry chain production. Journal of Advanced Veterinary Research, 7: 33-38.

Erfan AM and Marouf SH (2015). Biofilmproducing Staphylococcus aureus screening in poultry farms and abattoirs. Journal of Animal and Veterinary Advances, 14 (10): 273279.

Ferreira AM, Martins KB, Da Silva VR, Mondelli AL and Da Cunha MD (2017). Correlation of phenotypic tests with the presence of the blaZ gene for detection of beta-lactamase. Brazilian Journal of Microbiology, 48: 159-166.

Gao J, Ferreri M, Liu XQ, Chen LB, Su JL, Han B (2011). Development of multiplex polymerase chain reaction assay for rapid detection of Staphylococcus aureus and selected antibiotic resistance genes in bovine mastitic milk samples. Journal of Veterinary Diagnostic Investigation, 23(5): 894-901.

Götz, F., Bannerman, T. and Schleifer, K.L. (2006). The genera Staphylococcus and Macrococcus. In: The Prokaryotes, A Handbook on the biology of bacteria, Dworkin, M., Falkow, S., Rosenberig, E., Schleifer, K. and Stackebrands, E. (Eds.), $3^{\text {rd }}$ Ed., Springer Science+Business Media, LLC, pp: 5-75.

Grundmann H, Aanensen DM, Van Den Wijngaard CC, Spratt BG, Harmsen D, Friedrich AW (2010). Geographic distribution of Staphylococcus aureus causing invasive infections in Europe: a molecular-epidemiological analysis. PLoS medicine, 7:e1000215.

Hafez HM and Attia YA (2020). Challenges to the poultry industry: Current perspectives and strategic future after the COVID-19 outbreak. Frontiers in Veterinary Science, 7:516.

Kawano J, Shimizu A, Saitoh Y, Yagi M, Saito T, Okamoto R (1996). Isolation of methicillin-resistant coagulasenegative Staphylococci from chickens. Journal of Clinical Microbiology, 34 (9): 2072

Lowder BV, Guinane CM, Zakour NL, Weinert LA, Conway-Morris A, Robyn A, Cartwright RA, Simpson AJ, Rambaut A, Nübel U, Fitzgerald JR (2009). Recent human-to-poultry host jump, adaptation, and pandemic spread of Staphylococcus aureus. Proceedings of the National Academy of Sciences, 106: 19545-19550.

Magiorakos AP, Srinivasan A, Carey RB, Carmeli Y, Falagas ME, Giske CG, Harbarth S, Hindler JF, Kahlmeter G, Olsson-Liljequist B, Paterson DL, Rice LB, Stelling J, Struelens MJ, Vatopoulos A, Weber JT, Monnet DL (2012). Multidrug-resistant, extensively drug-resistant and pandrug-resistant bacteria: an international expert proposal for 
interim standard definitions for acquired resistance. Clinical Microbiology and Infection, 18: 268281.

Mamza SA, Geidam YA, Mshelia GD, Egwu GO (2019). Carriage, antibiotic susceptibility, and beta-lactamase production profiles of coagulasepositive Staphylococcus aureus isolated from chickens in NorthEastern Nigeria. Annals of Clinical Microbiology and Antimicrobials, 4(1): 1022.

Marek A, Stepień-Pyśniak D, Pyzik E, Wilczyński J, Winiarczyk S (2016). Occurrence and characterization of Staphylococcus bacteria isolated from poultry in Western Poland. Berliner und Munchener tierarztliche Wochenschrift, 129: 147-152.

Mason WJ, Blevins JS, Beenken K, Wibowo N, Ojha N, Smeltzer MS (2001). Multiplex PCR protocol for the diagnosis of Staphylococcal infection. Journal of Clinical Microbiology, 39 (9): 3332-3338.

McClure J-A, Conly JM, Lau V, Elsayed S, Louie T, Hutchins W, Zhang K (2006). Novel multiplex PCR assay for detection of the Staphylococcal virulence marker panton-valentine leukocidin genes and simultaneous discrimination of methicillinsusceptible from-resistant Staphylococci. Journal of Clinical Microbiology, 44: 1141-1144.

Melo PD, Ferreira LM, Filho AN, Zafalon LF, Vicente HI, Souza VD (2013). Comparison of methods for the detection of biofilm formation by Staphylococcus aureus isolated from bovine subclinical mastitis. Brazilian Journal of Microbiology, 44:119-124.
Mohamed FM, Bakheet AA, Othman AA, Mahmoud UT, Amen OA (2020). In vitro effect of zinc oxide nanoparticles on Staphylococcus aureus isolated from layer chickens. SVUInternational Journal of Veterinary Sciences, 3 (2):14-24.

Nasr RA, AbuShad HM and Hussein HS (2012). Biofilm formation and presence of icaAD gene in clinical isolates of Staphylococci. Egyptian Journal of Medical Human Genetics, 13: 269-274.

Onaolapo JA, Igwe JC, Bolaji RO, Adeshina GO, Parom SK (2017). Antibiotics susceptibility profile of Staphylococcus aureus isolated from poultry birds in Kaduna, Nigeria. Journal of Clinical Microbiology and Antimicrobials, 1: 101.

Otalu OJ, Kabir JJ, Okolocha EC, Umoh VJ (2011). Multi-drug resistant Staphylococcus aureus from live and slaughtered chickens in Zaria, Nigeria. International Journal of Poultry Science, 10: 871-875.

Otalu OJ, Kabir JJ, Okolocha EC, Umoh VP, Waga JK, and Owolodun AO (2015). Detection of methicillin-resistant Staphylococcus aureus in chicken carcasses and live birds in Zaria, Nigeria. FUTA Journal of Research in Science, 11: 132-138.

Palanisamy S and Bamaiyi PH (2015). Isolation and antibiogram of Salmonella spp. from quails in a farm from Kelantan, Malaysia. Journal of Veterinary Advances, 5 (12):11911198.

Patel R, Uhl JR, Kohner P, Hopkins MK, Cockerill FR (1997). Multiplex PCR detection of vanA, vanB, vanC-1, and vanC-2/3 denes in Enterococci. 
Journal of Clinical Microbiology, 35 (3): 703-707.

Popy N, Asaduzzaman M, Miah MS, Siddika A, Sufian MA, Hossain MM (2011). Pathological study on the upper respiratory tract infection of chickens and isolation, identification of causal bacteria. The Bangladesh Veterinarian, 28 (2): 60-69.

Pyzik E, Marek A, Stępień-Pyśniak D, Urban-Chmiel R, Jarosz ŁS, JagiełłoPodębska, I (2019). Detection of antibiotic resistance and classical enterotoxin genes in coagulasenegative Staphylococci isolated from poultry in Poland. Journal of Veterinary Research, 63:183-190.

Quinn, P.J., Carter, M.E., Markey, B. and carter G.R. (2004). Clinical Veterinary Microbiology. $1^{\text {st }}$ Ed., Mosby, Elsevier Limited, London.

Raidal, S. R. (1995). Staphylococcal Dermatitis in Quail with a Parakeratotic Hyperkeratotic Dermatosis Suggestive of Pantothenic Acid Deficiency. Avian Pathology 24 (3): 579-83.

Salah MA, Mohamed HMA, Abd Al-azeem MW (2020). Molecular detection of antibiotic resistance and virulence genes in Staphylococcus species isolated from human and poultry SVU-International Journal of Veterinary Sciences, 3(1): 100-122.

Shokry, Mariam, Ismail Redwan, Mohamed Amer, and Wafaa Ghany (2018). "Molecular Detection of Antibiotic Resistance Genes in Identified of Coagulase Negative Staphylococci from Chickens Flocks and Hatcheries in Egypt." Egyptian Journal of Veterinary Sciences 0 (0): 59-70.

Stepanović S, Vuković D, Veronika H, Bonaventura G, Djukić S, Ćirković I, Ruzicka F (2007). Quantification of biofilm in microtiter plates: Overview of testing conditions and practical recommendations for assessment of biofilm production by Staphylococci. Acta pathologica, microbiologica, et immunologica Scandinavica, 115 (8):891-899.

Stępień-Pyśniak D, Wilczyński J, Marek A, Śmiech A, Kosikowska U, Hauschild T (2017). Staphylococcus simulans associated with endocarditis in broiler chickens. Avian Pathology, 46: 44-51.

Vanessa Silva Madalena Vieira-Pinto , Cândido Saraiva Vera Manageir, Lígia Reis Eugénia Ferreira, Mo anuela Caniça José L. Capelo Gilberto Igrejas and Patrícia Poet (2021). Prevalence and Characteristics of MultidrugResistant Livestock-Associated Methicillin-Resistant Staphylococcus aureus (LA-MRSA) CC398 Isolated from Quails (Coturnix Coturnix Japonica) Slaughtered for Human Consumption. Animals, 11, 2038.

Vuong C and Otto M (2002). Staphylococcus epidermidis infections. Microbes and infection, 4:481-489.

White DG, Ayers S, Maurer JJ, Thayer SG, Hofacre C (2003). Antimicrobial susceptibilities of Staphylococcus aureus isolated from commercial broilers in Northeastern Georgia. Avian diseases, 47:203-210. 